\title{
ROLE OF LUNG POINT NAVIGATION BRONCHOSCOPY IN THE DIAGNOSIS OF PULMONARY NODULE
}

\author{
Mohamed Sedky El-Sayed ${ }^{1}$ MD, Yasser Ahmed El-Sayed ${ }^{2}$ MD, Fareed Shawky Basiony ${ }^{3}$ MD, Ali
} Samy Abdel-Khalik Ali" ${ }^{* *}$ MBBCH

\begin{abstract}
*Corresponding Author
Ali Samy Abdel-Khalik Ali

mradwan8892@yahoo.com

Received for publication January 31,

2020; Accepted May 2, 2020;

Published online May, 3, 2020

Copyright 2020 The Authors published by Al-Azhar University, Faculty of Medicine, Cairo, Egypt. All rights reserved. This an open-access article distributed under the legal terms, where it is permissible to download and share the work provided it is properly cited. The work cannot be changed in any way or used commercially.

doi: 10.21608/aimj.2020.23014.1111

${ }^{1}$ Department of Chest Disease, AlAzhar University, Cairo, Egypt

${ }^{2}$ Department of Cardiothoracic Surgery, Military Medical Academy, Cairo, Egypt.

${ }^{3}$ Chest hospital of Military Kobri AlKobba Medical Complex, Cairo, Egypt.
\end{abstract}

\begin{abstract}
Background: Pulmonary nodules are one of the most common thoracic imaging abnormalities. A revised estimate of over 1 million nodules is detected each year as an incidental finding, either on chest radiographs or thoracic computed tomography (CT) scans. However, an accurate diagnosis from these small, peripheral lung lesions can still be challenging with the use of conventional procedures. The lung point navigation bronchoscopy seems safe and effective. Navigation support with fluoroscopic guidance more successful in sampling small Solitary Pulmonary Nodules, increasing the likelihood of diagnosis, and decreasing the need for a surgical biopsy.

Aim of the work: To detect the role of the lung point navigation bronchoscopy in the diagnosis of the pulmonary nodule.

Patients and Methods: This study was performed between January 2019 and October 2019 in the bronchoscopy unit of Chest hospital of Military Kobri Al-Kobba medical Complex. It was a prospective cross-sectional analytic study conducted on 30 patients to detect the role of the lung point navigation system in the diagnosis of the pulmonary nodule.

Conclusion: Diagnosis of the pulmonary nodule by navigated bronchoscopy with the lung point system seems safe and effective. Navigation support with fluoroscopic guidance may be more successful in sampling small and peripherally located pulmonary nodules, increasing the likelihood of diagnosis, and decreasing the need for a surgical biopsy.
\end{abstract}

Keywords: Lung point navigation system; pulmonary nodule

Disclosure: The authors have no financial interest to declare in relation to the content of this article. The Article Processing Charge was paid for by the authors.

Authorship: All authors have a substantial contribution to the article

\section{INTRODUCTION}

Pulmonary nodules are one of the most common thoracic imaging abnormalities. A revised estimate of over 1 million nodules is detected each year as an incidental finding, either on chest radiographs or thoracic computed tomography (CT) scans ${ }^{1}$.

In lung cancer screening studies that enrolled people at high risk for lung cancer.

The prevalence of solitary pulmonary nodules ranged between $8-51 \%{ }^{2}$.

However, an accurate diagnosis from these small, peripheral lung lesions can still be challenging with the use of conventional procedures. Options available to diagnose newly identified lung lesions, include flexible fiberoptic bronchoscopy (FFB), CT-guided transthoracic biopsy, bronchoscopy with endobronchial ultrasound (EBUS) and thoracoscopic or open thoracic surgery. FOB is useful for visible endobronchial and central lesions, with an overall sensitivity of roughly $88 \% \%^{3}$.

While computerized-assisted transthoracic needle aspiration is one of the most frequently performed procedures, with the diagnostic yield approaching $65-90 \%$, it is limited due to its complications, in particular pneumothorax, requiring chest tube 
placement and hospitalization in almost half of the patients in some studies ${ }^{4}$.

Therefore to investigate and establish the diagnosis of suspicious lung lesions, particularly located beyond the subsegmental level in the lung, there is a need for a safer and more accurate procedure, not only for early diagnosis of the lesion with fewer complications but also to avoid unnecessary delay and surgical procedures. Virtual bronchoscopy is one such CT image-based technique that helps bronchoscopists to get to the lesion. Virtual bronchoscopy is a method for displaying 3dimensional images of the tracheal and bronchial lumens prepared from the continuous volume data of helical CT as if they have been observed on bronchoscopy ${ }^{5}$.

In Virtual Bronchoscopic Navigation, a bronchoscope is guided using Virtual Bronchoscopic images on the bronchial route to a Peripheral Pulmonary Lesion ${ }^{6}$.

Specific CT construction image parameters that provide good quality $3 \mathrm{D}$ volume, and subsequently detailed airway segmentation and virtual bronchoscopy, are under investigation and development. However, a CT that is appropriately collimated to thin slices $(0.5-1 \mathrm{~mm})$ and overlaps the image reconstruction is generally used to prepare the VB images down to the peripheral bronchi ${ }^{7}$.

There are two Virtual Bronchoscopic systems commercially available: the BF-NAVI system and the Lung Point system, the efficacy of VB is variable in the diagnosis of peripheral lung lesions. It has been reported in the range of $44.4-78.9 \% 8$.

Lung point navigation is a technique that is designed to guide the bronchoscopist to peripheral lesions based on Virtual Bronchoscopic images of the bronchial tree ${ }^{6}$.

Under direct visualization of the bronchial pathway to the lesion displayed on the Virtual Bronchoscopic images, this technique allows the bronchoscope to be readily guided to the target in a short time, irrespective of the bronchoscopist's skill level. Also, the systems that automatically search for the bronchial route to the target when the target is set to produce VB images on the route, and VB images matched with real images have been developed and clinically applied ${ }^{9}$.

Virtual Bronchoscopic Navigation has been used in conjunction with ultrathin bronchoscopy and endobronchial ultrasonography with a guide sheath (EBUS-GS), and excellent results have been reported ${ }^{10}$.

So, this study aimed to assess the lung point navigation bronchoscopy in the diagnosis of the pulmonary nodule.

\section{PATIENTS AND METHODS}

This study was performed between January 2019 and October 2019 in the bronchoscopy unit of Chest hospital of Military Kobri Al-Kobba Medical Complex, It was a prospective cross-sectional analytic study conducted on 30 patients to detect the role of the lung point navigation system in diagnosis of the pulmonary nodule.

Inclusion criteria: Pulmonary nodule or nodules on CT examination.

Exclusion criteria: Age years below 18 old, Accessible biopsies by fiber-optic bronchoscopy and presence of contraindications to bronchoscopy which are:

Absolute contraindications: Inadequate oxygenation during the procedure $(\mathrm{O} 2$ saturation $<90 \%$ on 2 $\mathrm{L} / \mathrm{min}$.) and recent angina or myocardial infarction. Relative contraindications: Untreatable lifethreatening arrhythmia, unstable cardiac status, refractory hypoxemia, bleeding diathesis or severe thrombocytopenia, uncooperative patient, unstable asthma and hypercapnia

Informed consent was obtained from all patients enrolled in the study.

All included patients were subjected to the following: History taking, clinical examination, laboratory investigations, and imaging studies: Plain chest x-ray posteroanterior view, CT scan of the chest using DICOM (Digital Imaging and Communications in Medicine) extension to be loaded on the lung point system.

Bronchoscopy procedure: The Lung Point Navigation Bronchoscopy is a computer-assisted image-based navigation software that enables the bronchoscopist to navigate and localize a targeted area of interest in the lung. With Lung Point, automatic processing is initiated upon input of CT information in a DICOM (Digital Imaging and Communications in Medicine) format into the system, after which the airway is extracted. Axial, sagittal, and coronal cross-sectional CT images are then displayed.

We defined the target lesion by placing a threedimensional spherical marker on a CT image.

The software automatically segments the object in three dimensions. Once the target object is placed, the software calculates the pathways through the airways to reach it.

During bronchoscopy, the navigation system displays two main images: the live bronchoscopic video and the VB animation, the animation represents the pathway that was selected during the planning process. An image-based synchronization technique aligns the virtual images with the anatomy seen in the live bronchoscopic video.

When the two images are synchronized, the software calculates the position of the bronchoscope in the airway and overlays the pathway to the target on the actual bronchoscopic video image.

Biopsy under general anesthesia was performed by a trained bronchoscopist using an ultrathin bronchoscope. After a complete inspection of the bronchial tree, including the subsegmental bronchi, the bronchoscope was navigated using Lung Point as near as possible to the target. After reaching the target, the procedure continued with transbronchial biopsy if no endobronchial lesion with using fluoroscopy. After taking the biopsy it was sent for histopathological examination.

The Used Equipment: The flexible bronchoscope used was Pentax EB-1830T3 video bronchoscope (5.0 $\mathrm{mm}$ insertion tube, $2.2 \mathrm{~mm}$ working channel, 60 $\mathrm{cm}$ working length[ and $3.7 \mathrm{~mm}$ insertion tube, 1.2 
mm working channel, $60 \mathrm{~cm}$ working length). The forceps and Lung point navigation bronchoscopy.

\section{RESULTS}

The mean age of the studied patients was 57.13 years; the male represents $66.7 \%$ and females represent $33.3 \%$.

\begin{tabular}{|c|c|c|}
\hline Variable & & $\mathbf{N}=\mathbf{3 0}$ \\
\hline \multirow{2}{*}{ Sex } & Female & $10(33.3 \%)$ \\
\cline { 2 - 3 } & Male & $20(66.7 \%)$ \\
\hline \multirow{2}{*}{$\begin{array}{c}\text { Age } \\
\text { years })\end{array}$} & Mean \pm SD & $57.13 \pm 11.95$ \\
\cline { 2 - 3 } & Range & $18-74$ \\
\hline
\end{tabular}

Table (1): Demographic characteristics of the studied patients

The most common sites of pulmonary nodules were right upper lobe, left upper lobe and left lower lobe $(23.3 \%, 23.3 \%, 20 \%)$ respectively.

\begin{tabular}{|c|c|c|c|}
\hline Variable & & $\mathbf{n}=\mathbf{3 0}$ & $\%$ \\
\hline \multirow{3}{*}{$\begin{array}{l}\text { Site of } \\
\text { nodule }\end{array}$} & LL & 6 & $20.0 \%$ \\
\hline & LU & 7 & $23.3 \%$ \\
\hline & RU & 7 & $23.3 \%$ \\
\hline \multirow{4}{*}{ Margin } & Irregular & 6 & $20.0 \%$ \\
\hline & Speculated & 10 & $33.3 \%$ \\
\hline & Lobulated & 3 & $10.0 \%$ \\
\hline & Smooth & 7 & $23.3 \%$ \\
\hline \multirow{5}{*}{ Calcification } & Central & 1 & $3.33 \%$ \\
\hline & Laminated & 2 & $6.7 \%$ \\
\hline & Popcorn & 0 & 0 \\
\hline & Diffuse & 2 & $6.7 \%$ \\
\hline & eccentric & 6 & $20 \%$ \\
\hline \multirow[t]{2}{*}{ Cavitation } & $\begin{array}{l}\text { Thin and } \\
\text { smooth wall }\end{array}$ & 3 & $10.0 \%$ \\
\hline & $\begin{array}{l}\text { Thick and } \\
\text { irregular wall }\end{array}$ & 2 & $6.7 \%$ \\
\hline \multirow{2}{*}{$\begin{array}{c}\text { Ground } \\
\text { glass nodule }\end{array}$} & Solid & 24 & $80 \%$ \\
\hline & Subsolid & 6 & $20 \%$ \\
\hline
\end{tabular}

Table (2): Character of the pulmonary nodule in CT of studied patients

Most of the pulmonary nodules in this study were malignant $(63.3 \%)$ while benign and non-specific inflammation were $(23.3 \%, 13.3 \%)$ respectively (Table 3).

\begin{tabular}{|c|c|c|c|}
\hline Variable & N & $\%$ \\
\hline \multirow{2}{*}{$\begin{array}{c}\text { Diagnosis } \\
\text { of nodules }\end{array}$} & Menign & 7 & $23.3 \%$ \\
\cline { 2 - 4 } & $\begin{array}{c}\text { Non specific } \\
\text { inflammation }\end{array}$ & 19 & $63.3 \%$ \\
\hline
\end{tabular}

Table (3): Types of nodules

The most diagnosed type of lung cancer after histopathology of biopsies taken via lung point navigation was Adenocarcinoma (AC) (43.3\%) followed by $\mathrm{SmCC}(10 \%)$ and Squamous cell carcinoma $(6.7 \%)$ (Table 4).

\begin{tabular}{|c|c|c|c|}
\hline Variable & Type & $\mathbf{N}$ & $\%$ \\
\hline \multirow{9}{*}{$\begin{array}{c}\text { Histopathological } \\
\text { Diagnosis }\end{array}$} & Adenocarcinoma (AC) & 13 & $\begin{array}{c}43.3 \\
\%\end{array}$ \\
\hline & $\begin{array}{l}\text { Mucoepidermoid } \\
\text { carcinoma (MEC) }\end{array}$ & 1 & $3.3 \%$ \\
\hline & $\begin{array}{l}\text { Small cell carcinoma } \\
\text { (SmCC) }\end{array}$ & 3 & $\begin{array}{c}10.0 \\
\%\end{array}$ \\
\hline & $\begin{array}{c}\text { Squamous cell } \\
\text { carcinoma }(\mathrm{SqCC})\end{array}$ & 2 & $6.7 \%$ \\
\hline & Fungal (aspergillosis) & 1 & $3.3 \%$ \\
\hline & Organizing pneumonia & 1 & $3.3 \%$ \\
\hline & Sarcoidosis & 2 & $6.7 \%$ \\
\hline & Tuberculosis (TB) & 3 & $\begin{array}{c}10.0 \\
\%\end{array}$ \\
\hline & $\begin{array}{l}\text { Nonspecific } \\
\text { inflammation }\end{array}$ & 4 & $\begin{array}{c}13.3 \\
\%\end{array}$ \\
\hline
\end{tabular}

Table (4): Histopathological diagnosis of nodules

Lung point navigation had succeeded in the diagnosis of $76.67 \%$ of studied cases and failed in the diagnosis of $23.33 \%$ of cases as shown in Table 5 .

\begin{tabular}{|c|c|c|}
\hline Navigation success & No. & $\%$ \\
\hline Success & 26 & $86.7 \%$ \\
\hline Failure & 4 & $13.3 \%$ \\
\hline
\end{tabular}

Table (5): Lung point navigation success in the studied patients

\section{DISCUSSION}

Lung Point combines a new virtual bronchoscopic navigation system and computer-assisted imagebased navigation software that enables physicians to navigate to localized areas of interest in the lung ${ }^{11}$. The use of navigation techniques can push the diagnostic yield of flexible bronchoscopy closer to the sensitivity obtainable with transthoracic CT guided procedures. Also, Pneumothorax, the major risk for transthoracic CT-guided procedures, can be dramatically reduced (from $20-30 \%$ to $3-5 \%)^{\mathbf{1 2}}$.

In our study, there was a highly statistically significant diagnostic yield of lung point navigation bronchoscopy in the diagnosis of pulmonary nodules. As recorded, all 30 patients, Lung point navigation had succeeded in the diagnosis of $86.66 \%$ of studied cases $(26 / 30)$ and failed in the diagnosis of $13.33 \%$ of cases (4/30).

Our study agrees with that done by Eberhardt et al in 25 patients (9 women and 16 men), generally, a diagnosis was established in 20 of the 25 subjects $(80 \%)^{13}$.

Also, in Tamiya et al study The diagnostic yield for thin bronchoscopy with EBUS-GS under Lung Point Navigation guidance for small $(<30 \mathrm{~mm})$ PPL was $77.9 \%{ }^{14}$.

Most of the pulmonary nodules in this study were malignant $(63.3 \%)$ in 19 patients while 7 patients were diagnosed as benign while 4 patients had nonspecific inflammation $(23.3 \%, 13.3 \%)$ respectively.

These results agree with those of Eberhardt et al who reported that among the 20 subjects in whom biopsy was diagnostic, 17 of the lesions were classified as malignant and three lesions were classified as benign 13 . 
Regarding histopathological examination for tissue biopsies in our study $(\mathrm{n}=30), 13$ patients $(43.3 \%)$ were diagnosed adenocarcinoma. 3 patients $(10 \%)$ were diagnosed as small cell carcinoma, 2 cases had squamous cell carcinoma $(6.7 \%), 2$ cases had Sarcoidosis, 1 case had Mucoepidermoid carcinoma, 1 case had organizing pneumonia, 1 case of Fungal pool, and 3 cases had Tuberculosis (TB). These results agree with a study done by Eberhardt et $\boldsymbol{a l} \mathbf{l}^{13}$ who reported that Among the 20 subjects in whom biopsy was diagnostic, 17 of the lesions were classified as malignant (11 cases of adenocarcinoma, 3 cases of Squamous cell carcinoma, 1 case of nonsmall cell lung cancer, 1 case of small cell lung cancer, and 1 case of carcinoma with large cells). Of the diagnostic biopsies, three lesions were classified as benign (one Sarcoidosis, one Tuberculoma, and one rheumatic nodule) ${ }^{\mathbf{1 3}}$.

Our results also came against those of Biswas et al ${ }^{15}$ who reported that Squamous cell cancer account for approximately $25 \%$ of studied cases (13 cases), adenocarcinoma is about $23.07 \%$ (12 cases), small cell cancer and carcinoid tumor about $3.84 \%$ ( 2 cases for each), while six cases diagnosed as benign ( 3 cases of non-tuberculous mycobacterium, one each of Moraxella and Pneumococcal and one Aspergillus pneumonia ${ }^{15}$

Lung point navigation bronchoscopy shortened the total examination time, especially the time to sample collection. The duration of X-ray fluoroscopy was also shortened. Asano et al performed a multicenter randomized controlled study on transbronchial lung biopsy of 3-cm or smaller peripheral lesions using an ultrathin bronchoscope, in which VBN increased the rate of biopsy instrument arrival at the lesion ${ }^{\mathbf{1 6}}$.

In the present study, the mean planning time was 5 minutes (SD 0.3 seconds; range, 4.5-5.5 minutes). The total examination time was 21 minutes (SD_2.9 minutes; range, 16-25 minutes). The mean Lung Point navigation time was 7.4 minutes (SD _ 0.8 minutes; range, 5.6-9 minutes).

Our study came against that done by Asano et al revealed that the Total examination time was 16.6 minutes (range, 7.6-36.5 minutes). The mean examination time before sample collection was 5.9 minutes (range, 2.2-18.2 minutes). ${ }^{16}$

The diagnostic yield in our study was $100 \%$ for the RUL and LUL, 66.67 for LLL, $50 \%$ for combined RLL and LLL, and $33.33 \%$ for RLL. A lesion in the RUL was more likely to have a diagnosis $(17 / 21$; $81 \%$ ) in contrast to all other lobes combined (18/31; $58 \%$ ).

Our results came in agreement with those of Biswas et $\boldsymbol{a l}$. which revealed that the diagnostic yield was $81 \%, 61 \%, 37 \%, 66 \%$, and $75 \%$ for the RUL, LLL, LUL, RML, and RLL. A lesion in the RUL was more likely to have a diagnosis $(17 / 21 ; 81 \%)$ in contrast to all other lobes combined $(18 / 31 ; 58 \%){ }^{1}$

In the current study, there was a highly significant relationship between the size of nodules and the diagnosis. As shown in the study that the mean size of $27 \mathrm{~mm}$ was significantly diagnosed, while the mean size of 16.5 was undiagnosed $(\mathrm{p}=0.001)$.

We have demonstrated in the current study the successful application of lung point navigation bronchoscopy guided ultrathin bronchoscopy in sampling peripheral lung lesions; there was a highly statistically significant diagnostic yield of the lung point navigation system in the diagnosis of the pulmonary nodule.

\section{CONCLUSION}

The diagnosis of the pulmonary nodule by navigated bronchoscopy with the lung point system seems safe and effective. Navigation support with fluoroscopic guidance may be more successful in sampling small and peripherally located pulmonary nodules, increasing the likelihood of diagnosis, and decreasing the need for a surgical biopsy.

Funding: No funding sources.

Conflict of interest: None declared.

Acknowledgment: All the staff members of Chest Department, Al-Azhar University, Cairo, and Military Medical Academy, Cairo during the study. Ethical approval: The study was approved by the Institutional Ethics Committee.

\section{REFERENCES}

1. Gould M, Tang T, Liu L, et al. Recent trends in the identification of incidental pulmonary nodules. American Journal of Respiratory and Critical Care Medicine, 2015; 192(10): 1208-1214.

2. Wahidi M, Govert J, Goudar R, et al. Evidence for the treatment of patients with pulmonary nodules: when is it lung cancer? ACCP evidence-based clinical practice guidelines. Chest, 2007;132(3): $94-$ 107.

3. Rivera M, Mehta A and Wahidi M. Establishing the diagnosis of lung cancer: Diagnosis and management of lung cancer: American College of Chest Physicians evidence-based clinical practice guidelines. Chest, 2013; 143(5): 142-165.

4. Khan K, Zaidi S and Swan N. The use of computerized tomography guided percutaneous fine needle aspiration in the evaluation of solitary pulmonary nodules. Ir Med J. 2012;105(2):50-52.

5. Vining D, Liu K, Choplin R, et al. Virtual bronchoscopy: relationships of virtual reality endobronchial simulations to actual bronchoscopic findings. Chest, 1996; 109(2): 549-553.

6. Asano $\mathrm{F}$, Matsuno $\mathrm{Y}$, Matsushita $\mathrm{T}$, et al. Transbronchial diagnosis of a pulmonary peripheral small lesion using an ultrathin bronchoscope with virtual bronchoscopic navigation. Journal of Bronchology \& Interventional Pulmonology,2002; 9(2): 108-111.

7. Nardelli P, Khan K, Corvò A, et al. Optimizing parameters of an open-source airway segmentation algorithm using different CT images. Biomedical engineering online, 2015; 14(1): 62 .

8. Asano F, Eberhardt R and Herth FJ. Virtual bronchoscopic navigation for peripheral pulmonary lesions. Respiration, 2014; 88(5): 430-440.

9. Asano F, Matsuno Y, Tsuzuku A, et al: Diagnosis of peripheral pulmonary lesions using a bronchoscope insertion guidance system combined with endobronchial ultrasonography with a guide sheath. Lung Cancer, 2008; 60(3): 366-373. 
10. Shinagawa N, Yamazaki K, Onodera Y, et al. Virtual bronchoscopic navigation system shortens the examination time-feasibility study of virtual bronchoscopic navigation system. Lung Cancer, 2007; 56(2): 201-206.

11. Rivera, M and Mehta, A. Initial diagnosis of lung cancer: ACCP evidence-based clinical practice guidelines. Chest, 2007; 132(3), 131S-148S.

12. Yoshikawa M, Sukoh N, Yamazaki, K, et al. Diagnostic value of endobronchial ultrasonography with a guide sheath for peripheral pulmonary lesions without X-ray fluoroscopy. Chest,2007; 131(6), 1788-1793.

13. Eberhardt R, Kahn N, Gompelmann D, Schumann M, et al. Lung Point-A New Approach to Peripheral Lesions, J Thorac Oncol, 2010; 5, 1559-1563.

14. Tamiya M, Okamoto N, Sasada $\mathrm{S}$ et al. Diagnostic yield of combined bronchoscopy and endobronchial ultrasonography, under Lung Point guidance for small peripheral pulmonary lesions. Respirology, 2013;18, 834-839

15. Biswas A, Mehta H and Sriram P. Diagnostic Yield of the Virtual Bronchoscopic Navigation System Guided Sampling of Peripheral Lung Lesions using Ultrathin Bronchoscope and Protected Bronchial Brush. Turk Thorac J, 2019; 20(1): 6-11.

16. Asano F, Shinagawa N, Ishida T, et al. Virtual bronchoscopic navigation combined with ultrathin bronchoscopy. A randomized clinical trial. American Journal of Respiratory and Critical Care Medicine, 2013;188(3), 327-333. 